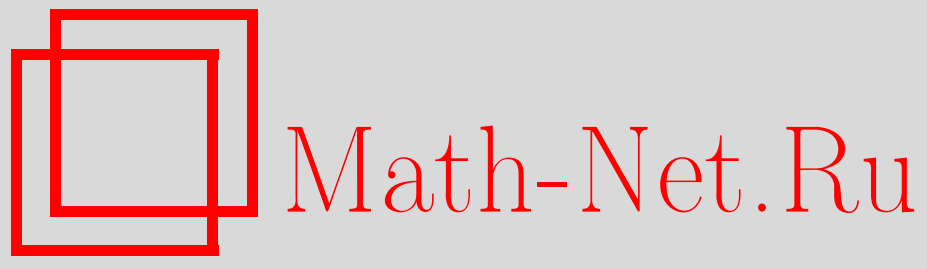

А. Л. Якымив, Тауберовы теоремы и асимптотика безгранично делимых распределений в конусе, Теория вероятн. и ее примен., 2003, том 48, выпуск $3,487-502$

DOI: https://doi.org/10.4213/tvp267

Использование Общероссийского математического портала MathNet.Ru подразумевает, что вы прочитали и согласны с пользовательским соглашением

http://www . mathnet.ru/rus/agreement

Параметры загрузки:

IP : 54.164 .48 .24

26 апреля 2023 г., 13:51:14

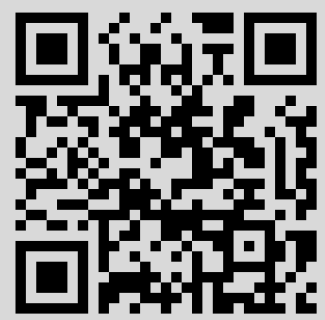




\title{
ТАУБЕРОВЫ ТЕОРЕМЫ И АСИМПТОТИКА БЕЗГРАНИЧНО ДЕЛИМЫХ РАСПРЕДЕЛЕНИЙ В КОНУСЕ ${ }^{1)}$
}

\begin{abstract}
В работе доказаны три многомерные тауберовы теоремы, при помощи которых установлена асимптотика на бесконечности безгранично делимых распределений с носителем в произвольном замкнутом выпуклом остром телесном однородном конусе из $\mathbf{R}^{n}$.

Ключевые слова и фразы: правильно меняющиеся функции вдоль семейства операторов, вполне допустимые функции для семейства операторов, вполне допустимые функции для конуса, безгранично делимые распределения, спектральная мера Леви.
\end{abstract}

1. Введение. В настоящей статье доказаны три многомерные тауберовы теоремы сравнения типа [7] в произвольном замкнутом выпуклом остром телесном конусе $\Gamma$ (см. п. 2, теоремы 1-3). Метод доказательства этих тауберовых теорем является синтезом методов упомянутой работы Ю.Н. Дрожжинова и Б. И. Завьялова [7] и работы автора [17]. При помощи этих тауберовых теорем получена асимптотика безгранично делимых распределений, сосредоточенных в конусе Г (см. п. 3 , теорема 4). Теорема 4 продолжает исследования асимптотики многомерных безгранично делимых распределений, проводившиеся ранее в работах В.М. Круглова и его ученика С.Н. Антонова [1]-[3], [8]-[13], М. С. Сгибнева [14], А. М. Улановского [15], Э. Омея [20] и автора [21]. Отметим, что проблеме изучения асимптотики безгранично делимых распределений на бесконечности посвяшено большое число работ (особенно в одномерном случае). Ряд ссылок имеется в статье [2], а также в статье [14]. Некоторые вопросы асимптотики многомерных безгранично делимых распределений на бесконечности затронуты в книге Кен-Ити Сато 1999 г. [19].

2. Многомерные тауберовы теоремы. Пусть $Г-$ замкнутый выпуклый острый телесный конус в $\mathbf{R}^{n}$ с вершиной в нуле, т.е. замкнутое выпуклое множество в $\mathbf{R}^{n}$ такое, что для всех $x \in \Gamma$ и $t>0 t x \in \Gamma$,

* Математический институт им. В. А. Стеклова РАН, ул. Губкина, 8, 119991 Москва, ГСП-1, Россия; e-mail: arsen@mi.ras.ru

1) Работа выполнена при финансовой поддержке РФФИ, грант 02-01-01080 и гранта президента РФ поддержки ведуших научных школ НШ 1758.2003.1. 
причем int $\Gamma \neq \varnothing$ и $\operatorname{int} \Gamma^{*} \neq \varnothing$, где

$$
\Gamma^{*}=\left\{y: y \in \mathbf{R}^{n},(y, x) \geqslant 0 \forall x \in \Gamma\right\} .
$$

При этом сопряженный конус тоже будет замкнутым выпуклым острым телесным. Более подробную информацию о конусах можно почерпнуть в книге В.С. Владимирова, Ю.Н. Дрожжинова и Б. И. Завьялова [5]. Положим $G=\operatorname{int} \Gamma, C=\operatorname{int} \Gamma^{*}$.

Пусть задано произвольное семейство $U=\left\{U_{k}, k \in I \subseteq[0, \infty)\right\}$ линейных операторов в $\mathbf{R}^{n}$, которые оставляют конус $\Gamma$ инвариантным:

$$
U_{k} \Gamma=\Gamma \quad \forall k \in I, \quad J_{k}=\operatorname{det} U_{k} .
$$

Мы будем считать, что множество $I$ имеет $\infty$ своей предельной точкой. Oператор $V_{k}=\left(U_{k}^{*}\right)^{-1}$ оставляет сопряженный конус $\Gamma^{*}$ инвариантным. Для произвольного оператора $U_{k}$ мы имеем числа

$$
\Lambda(k)=\sup _{|e|=1}\left|U_{k} e\right|, \quad \lambda(k)=\inf _{|e|=1}\left|U_{k} e\right| .
$$

Оператору $V_{k}$ будут соответствовать числа

$$
\frac{1}{\lambda(k)}=\sup _{|e|=1}\left|V_{k} e\right|, \quad \frac{1}{\Lambda(k)}=\inf _{|e|=1}\left|V_{k} e\right| .
$$

О п р е д е л н и е 1. Мы будем говорить, что семейство $U_{k}$, $k \in I$, является семейством: первого типа, если $\Lambda(k) \rightarrow \infty$ и $\lambda(k) \rightarrow \infty$ при $k \rightarrow \infty, k \in I$; второго типа, если существует $b>0$ такое, что $\Lambda(k) \rightarrow \infty$ при $k \rightarrow \infty, k \in I$ и $\lambda(k) \geqslant b>0(k \in I)$; третьего типа, если $\Lambda(k) \rightarrow \infty$ при $k \rightarrow \infty, k \in I$.

О п ре де ле н и е 2. Мы будем говорить, что функция $f(x)$, определенная, положительная и измеримая в $G$, правильно меняется в $G$ вдоль семейства $U=\left\{U_{k}, k \in I\right\}$, и писать $f \in R(U, G)$, если для некоторого вектора $e \in G$ и для всех $x \in G$ при $x_{k} \rightarrow x(k \rightarrow \infty, k \in I)$

$$
\frac{f\left(U_{k} x_{k}\right)}{f\left(U_{k} e\right)} \rightarrow \phi(x)>0, \quad \phi(x)<\infty .
$$

Из (1) следует (см. [18, теорема 2]), что

$$
\frac{f\left(U_{k} x\right)}{f\left(U_{k} e\right)} \stackrel{x \in K}{\rightrightarrows} \phi(x) \in(0, \infty) \quad(k \rightarrow \infty, k \in I)
$$

для произвольного компакта $K \subset G$, причем $\phi(x)$ непрерывна в $G$. Coгласно (3), мы будем записывать $\phi=H_{e}(U, G)$.

3 а м е ч н и е 1 . Множество $R(U, G)$ не зависит от вектора $e \in G$. При этом, если (3) выполнено для некоторого вектора $e \in G$, то оно будет выполнено для произвольного вектора $e_{1} \in G$, при этом функция $\phi$ умножится на $\lim _{k \rightarrow \infty, k \in I} f\left(U_{k} e\right) / f\left(U_{k} e_{1}\right)$. 
О п р е д е л е н и е 3 . Функцию $f(x)$, определенную в $G$, мы будем называть вполне допустимой для семейства операторов $U=\left\{U_{k}\right.$, $k \in I\}$, если $f \in R(U, G), f$ локально суммируема в $G$ и суцествует $k_{0}$ такое, что

$$
\frac{f\left(U_{k} x\right)}{f\left(U_{k} e\right)} \leqslant \eta(x), \quad k>k_{0}, \quad k \in I, \quad x \in G,
$$

где $e$ - некоторый фиксированный вектор из $G$, причем $\eta$ имеет медленный рост в $G$ :

$$
\int_{G} \frac{\eta(x) d x}{1+|x|^{q}}<\infty \quad \text { для некоторого } q .
$$

3 а м е ч а н и е 2. Определение 3 эквивалентно определению 0-вполне допустимой функции для семейства $U=\left\{U_{k}, k \in I\right\}$ из $[5$, гл. II, раздел 5.2].

О п р е д е л е н и е 4 . Функция $f(x)$, определенная в $G$, называется допустимой для конуса $\Gamma$, если для произвольного семейства линейных операторов $U=\left\{U_{k}, k \in I\right\}$, оставляющих конус $\Gamma$ инвариантным, существует подсемейство $V=\left\{U_{k}, k \in J \subseteq I\right\}$ ( $J$ - неограничено), относительно которого $f(x)$ будет вполне допустимой.

Множество всех допустимых функций для конуса $\Gamma$ обозначим через $D(\Gamma)$.

О п р е д е л е н и е 5 . Функция $f(x)$, определенная в $G$, называется допустимой $m$-го типа $(m=1,2,3)$ для конуса $\Gamma$, если для произвольного семейства линейных операторов $m$-го типа $U=\left\{U_{k}, k \in I\right\}$, оставляющих конус $\Gamma$ инвариантным, существует подсемейство $V=$ $\left\{U_{k}, k \in J \subseteq I\right\}$ ( $J$ - неограничено), относительно которого $f(x)$ будет вполне допустимой.

Множество всех допустимых функций $m$-го типа для конуса $\Gamma$ обозначим через $D_{m}(\Gamma), m=1,2,3$.

О б о з н а ч е н и е 1 . Через $R_{m}(\Gamma), m=1,2,3$, мы обозначим множество всех функций $f(x)$, определенных в $G$, таких, что для произвольного семейства линейных операторов $m$-го типа $U=\left\{U_{k}, k \in I\right\}$, оставляющих конус $\Gamma$ инвариантным, существует подсемейство $V=\left\{U_{k}, k \in\right.$ $J \subseteq I\}$ ( $J$ - неограничено) такое, что $f \in R(V, \Gamma)$ (см. определение 2).

О бо з н а ч е н и е 2 . Через $R(\Gamma)$ мы обозначим множество всех функций $f(x)$, определенных в $G$, таких, что для произвольного семейства линейных операторов $U=\left\{U_{k}, k \in I\right\}$, оставляющих конус $\Gamma$ инвариантным, сушествует подсемейство $V=\left\{U_{k}, k \in J \subseteq I\right\}$ ( $J$ - неограничено) такое, что $f \in R(V, \Gamma)$ (см. определение 2).

Ясно, что

$$
D(\Gamma) \subseteq D_{3}(\Gamma) \subseteq D_{2}(\Gamma) \subseteq D_{1}(\Gamma), \quad R(\Gamma) \subseteq R_{3}(\Gamma) \subseteq R_{2}(\Gamma) \subseteq R_{1}(\Gamma) .
$$


Приведем примеры функций из $\mathbf{R}(\Gamma)$ и $D(\Gamma)$.

Пусть $\mathbf{R}_{+}^{n}=\left\{x=\left(x_{1}, \ldots, x_{n}\right) \in \mathbf{R}^{n}, x_{i} \geqslant 0 \forall i=1, \ldots, n\right\}-$ положительный координатный угол (октант), $V_{n}^{+}=\left\{x=\left(x_{0}, x_{1}, \ldots, x_{n}\right) \in\right.$ $\left.\mathbf{R}^{n+1}, x_{0} \geqslant \sqrt{x_{1}^{2}+\cdots+x_{n}^{2}}\right\}$ - световой конус будущего в $\mathbf{R}^{n+1}$.

П р и м е р 1 (см. [5, гл. II, раздел 5.3, теорема 1]). Пусть $f(x)-$ положительная, непрерывно дифференцируемая функция в int $\mathbf{R}_{+}^{n}$, удовлетворяющая условию

$$
a \leqslant \frac{x_{j} \partial f(x) / \partial x_{j}}{f(x)} \leqslant b, \quad x_{j}>0, \quad j=1, \ldots, n
$$

при некоторых $a, b \in(-\infty, \infty)$. Тогда $f \in R\left(\mathbf{R}_{+}^{n}\right)$. В частности, если $a>-1$, то $f \in D\left(\mathbf{R}_{+}^{n}\right)$.

П р и м е р 2 (см. [5, гл. II, раздел 5.3, теорема 2]). Пусть $f(x)-$ положительная, непрерывно дифференцируемая функция в $\operatorname{int} V_{n}^{+}$и

$$
a \leqslant \frac{(l, x)(l, \nabla f(x))}{f(x)} \leqslant b, \quad x \in V_{n}^{+}, \quad l \in \partial V_{n}^{+}, \quad|l|=1,
$$

при некоторых $a, b \in(-\infty, \infty)$, где $\nabla=\left(\partial / \partial x_{1}, \ldots, \partial / \partial x_{n}\right)$. Тогда $f \in$ $R\left(V_{+}^{n}\right)$. В частности, если $a>-1$, то $f \in D\left(V_{n}^{+}\right)$.

Пусть $\Gamma$ - замкнутый выпуклый острый телесный конус в $\mathbf{R}^{n} \mathrm{c}$ вершиной в нуле. Рассмотрим функцию $\chi$, представимую в виде

$$
\chi(t)=\eta(t) \phi(t), \quad t \in(0, \infty),
$$

где функции $\eta$ и $\phi$ удовлетворяют условиям:

1) существуют положительные числа $c_{1}$ и $c_{2}$ такие, что

$$
c_{1} \leqslant \eta(t) \leqslant c_{2}, \quad t \in(0, \infty)
$$

2)

$$
\omega(\lambda, t)=\frac{\eta(\lambda t)}{\eta(t)} \stackrel{t \in(0, \infty)}{\rightrightarrows} 1, \quad \lambda>1 ;
$$

3) функция $\phi$ положительна, непрерывно дифференцируема и удовлетворяет неравенствам

$$
a \leqslant \frac{t \phi^{\prime}(t)}{\phi(t)} \leqslant b \quad(0<t<\infty)
$$

при некоторых $a, b \in(-\infty, \infty)$.

П р и м е р 3 (см. [5, гл. II, раздел 5.4]). Пусть функция $\chi$ удовлетворяет условиям (6)-(9) и фиксированы векторы $l_{i} \in \Gamma^{*}$, числа $A_{i}>0$ и $\alpha \in \mathbf{R}^{1}$. Тогда $f(x)=\chi(\omega(x)) \in R(\Gamma)$, где

$$
\omega(x)=\frac{\sum_{i=1}^{p} A_{i}\left(l_{i}, x\right)^{\alpha_{i}}}{\sum_{i=p+1}^{p+q} A_{i}\left(l_{i}, x\right)^{\alpha_{i}}}, \quad x \in \Gamma .
$$

В частности, если $-\alpha_{i} a<1$ при $i=1, \ldots, p$ и $\alpha_{i} b<1$ при $i=p+$ $1, \ldots, p+q$, то $f(x) \in D(\Gamma)$. 
Сформулируем некоторые необходимые сведения из [17] (подробные доказательства имеются в [16]). Пусть, как и ранее, $\Gamma$ - замкнутый выпуклый телесный конус в $\mathbf{R}^{n}$ с вершиной в нуле. В конусе $\Gamma$ введем отношение порядка [4]: будем писать, что

$$
x \leqslant y \quad(x \stackrel{\Gamma}{<} y),
$$

если $x, y, y-x \in \Gamma$ (соответственно $x \in \Gamma, y, y-x \in G)$. Действительную функцию $f(x)$, определенную в $G$, назовем неубывающей (соответственно невозрастающей) в $G$, если при $x, y \in G, x \stackrel{\Gamma}{<} y$ имеет место неравенство $f(x) \leqslant f(y)$ (соответственно $f(x) \geqslant f(y)$ ). В [16] доказана следующая лемма.

Лемма 1. Пусть $f$ не убывает в $G$ (не возрастает в $G)$.

1. Тогда для каждого $x \in G$ существуют два следующих предела:

$$
\lim _{y \uparrow x} f(y) \equiv \lim _{y \rightarrow x, y<x} f(y)=f\left(x_{-}\right), \quad \lim _{y \downarrow x} f(y) \equiv \lim _{y \rightarrow x, y>x} f(y)=f\left(x_{+}\right),
$$

где $f\left(x_{-}\right)=\sup (f(y), y \stackrel{\Gamma}{<} x), f\left(x_{+}\right)=\inf (f(y), y \stackrel{\Gamma}{>} x)$ (соответственно $\left.f\left(x_{-}\right)=\inf (f(y), y \stackrel{\Gamma}{<} x), f\left(x_{+}\right)=\sup (f(y), y \stackrel{\Gamma}{>} x)\right)$, причем $f\left(x_{-}\right) \leqslant$ $f(x) \leqslant f\left(x_{+}\right)$(соответственно $f\left(x_{-}\right) \geqslant f(x) \geqslant f\left(x_{+}\right)$).

2. Для того итобы $f$ была непрерывной в $x \in G$, необходимо $u$ достаточно, итобы $f\left(x_{-}\right)=f\left(x_{+}\right)$.

О б о з н а че н и е 3 . Через $M_{1}(G)$ (соответственно через $M_{2}(G)$ ) обозначим множество всех неубывающих (соответственно невозрастающих функций в $G$ ), непрерывных «сверху», т.е. таких, что для всех $x \in G f(x)=f\left(x_{+}\right), M(G)=M_{1}(G) \cup M_{2}(G)$.

Пусть $i=1$ или $i=2$. Будем говорить, что семейство $\left\{f_{k}, k \in I\right\}$, $f_{k} \in M_{i}(G)$, слабо сходится при $k \rightarrow \infty, k \in I$ к $f \in M_{i}(G)$, и писать $f_{k} \Rightarrow f(k \rightarrow \infty, k \in I)$, если $f_{k}(x) \rightarrow f(x)$ при $k \rightarrow \infty, k \in I$ для произвольной точки $x$ непрерывности функции $f$.

Пусть $\mathfrak{A}$ - совокупность всех ограниченных борелевских множеств из $\mathbf{R}^{n}$. Меры, рассматриваемые далее, будут считаться неотрицательными, $\sigma$-конечными и сосредоточенными на $\Gamma$. Мы будем говорить, что семейство мер $\mu_{k}, k \in I$, слабо сходится при $k \rightarrow \infty, k \in I$ к мере $\mu$, и писать $\mu_{k} \Rightarrow \mu(k \rightarrow \infty, k \in I)$, если $\mu_{k}(A) \rightarrow \mu(A)(k \rightarrow \infty, k \in I)$ для каждого $A \in \mathfrak{A}$ такого, что $\mu(\partial A)=0$. Совокупности монотонных функций или мер мы будем называть слабо предкомпактными, если они предкомпактны в определенных выше слабых топологиях. В [16] доказана следуюшая лемма.

Лемма 2. Пусть $i=1$ или $i=2$. Для того чтобы семейство функций $F \subseteq M_{i}(G)$ было слабо предкомпактным, необходимо и достаточно, чтобь $\sup _{f \in F}|f(x)|<\infty$ для каждого $x \in G$. 
Преобразования Лапласа меры $\mu$ и функции $f$ на Г будем обозначать соответственно через $\hat{f}(y)$ и $\tilde{\mu}(y)$ :

$$
\widetilde{\mu}(y)=\int_{\Gamma} e^{-(y, x)} \mu(d x), \quad \widehat{f}(y)=\int_{\Gamma} e^{-(y, x)} f(x) d x
$$

в предположении, что они существуют при $y \stackrel{T}{>} a$ для некоторого $a \in$ $T=\Gamma^{*}$.

Лемма 3. Пусть задано семейство мер $\mu_{k}, k \in I$.

1. Если для некоторого а $\in$ T существуют их преобразования Лаnласа $\tilde{\mu}_{k}(y), y \stackrel{T}{>} a, u$

$$
\tilde{\mu}_{k}(y) \rightarrow \omega(y)<\infty \quad(k \rightarrow \infty, k \in I)
$$

для каждого $у \stackrel{T}{>}$ a, то $\omega(y)$ является преобразованием Лапласа некоторой меры $\mu$ на Ги

$$
\mu_{k} \Rightarrow \mu \quad(k \rightarrow \infty, k \in I) .
$$

2. Обратно, если выполнено (11) и $\tilde{\mu}_{k}($ a) ограничено, то выполнено соотношение (10), где $\omega(y)=\tilde{\mu}(y)<\infty$ nри $y \stackrel{T}{>}^{T} a$.

3 а м е ч а н и е 3 . Лемма 3 доказана в [16] для случая $I=\mathbf{N}$, перенесение ее для произвольного $I$ дословно повторяет это доказательство.

Пусть $\Gamma$ - произвольный замкнутый выпуклый острый телесный конус, допускающий семейство линейных преобразований $U=\left\{U_{k}\right.$, $k \in I\}$, которые оставляют $\Gamma$ инвариантным (см. (1)). Справедлива следующая тауберова теорема.

Tеорема 1. Пусть $f(x)=u(x) v(x)(x \in G)$, где $u(x) \in R(U, G)$ (см. определение 2$), v(x) \in M(G)$ (см. обозначение 3 после леммы 1$)$, $v(x) \geqslant 0(x \in G)$, иисла $\rho_{k}>0(k \in I) u$ для всех $y \in \mathbf{C}$ cyuествует $\widehat{f}(y)$. Предположим, что для всех $y \in \mathbf{C}$

$$
\frac{\widehat{f}\left(V_{k} y\right)}{J_{k} \rho_{k}} \rightarrow \psi(y)<\infty \quad(k \rightarrow \infty, k \in I)
$$

$\left(V_{k}=\left(U_{k}^{*}\right)^{-1}, J_{k}=\operatorname{det} U_{k}\right)$. Тогда существуют определенная в $G$ функuия $\phi(x)<\infty(x \in G)$ такая, uто

$$
\frac{f\left(U_{k} x\right)}{\rho_{k}} \rightarrow \phi(x) \quad(k \rightarrow \infty, k \in I)
$$

почти всюду в $G$, и мера $\mu$ на Г такая, ито ф является ее плотностью ${ }_{8} G u$

$$
\psi(y)=\tilde{\mu}(y)<\infty \quad \forall y \in \mathbf{C} .
$$


В частности, если $\mu(\partial \Gamma)=0$, то

$$
\psi(y)=\widehat{\phi}(y)<\infty \quad \forall y \in \mathbf{C} .
$$

Если функиия $\phi(x)$ непрерывна в $G$, то соотношение (13) выполнено равномерно по $x \in K$ для произвольного компакта $K \subset G$.

3 а м е ч а н и е 4. Теорема 1 является аналогом теоремы 1 из [5, гл. II, раздел 4.3]. Основное ее отличие от цитируемой теоремы состоит в ослаблении тауберова условия и в отказе от предположения о регулярности конуса $\Gamma$.

Доказательство те оремы 1. Зададим семейство мер $\left\{\mu_{k}, k \in I\right\}$ на $\mathfrak{A}$ равенством

$$
\mu_{k}(A)=\int_{A \cap \Gamma} \frac{f\left(U_{k} x\right)}{\rho_{k}} d x, \quad A \in \mathfrak{A} .
$$

Так как

$$
\tilde{\mu}_{k}(y)=\frac{\widehat{f}\left(V_{k}(y)\right)}{J_{k} \rho_{k}} \quad(y \in \mathbf{C}),
$$

то из (12) следует, что для произвольного $y \in \mathbf{C}$

$$
\tilde{\mu}_{k}(y) \rightarrow \psi(y)<\infty \quad(k \rightarrow \infty, k \in I) .
$$

Согласно лемме 3 , для некоторой меры $\mu$ на $\Gamma$

$$
\mu_{k} \Rightarrow \mu \quad(k \rightarrow \infty, k \in I)
$$

причем выполнено соотношение (14). Зафиксируем произвольный вектор $e \in G$. Положим при $x \in G$

$$
u_{k}(x)=\frac{u\left(U_{k} x\right)}{u\left(U_{k} e\right)}, \quad v_{k}(x)=\frac{v\left(U_{k} x\right)}{\rho_{k}} u\left(U_{k} e\right) .
$$

Предположим для определенности, что $v \in M_{2}(G)$. Заметим, что для произвольного линейного оператора $U$, сохраняющего конус $\Gamma$ инвариантным $(U \Gamma=\Gamma)$,

$$
v(U x) \in M_{2}(G) \text {. }
$$

В самом деле, так как $U \Gamma=\Gamma$, то $U(\partial \Gamma)=\partial \Gamma$ и $U(G)=G$. Поэтому при $x, y \in G$ и $x \stackrel{\Gamma}{<} y$ имеем $U x, U y, U(y-x) \in G$, так как $y-x \in G$. Стало быть, $U x \stackrel{\Gamma}{<} U y$, значит, $v(U x) \geqslant v(U y)$. Далее, согласно лемме 1 ,

$$
\lim _{y \rightarrow x, y>x} v(U y)=\lim _{U y \rightarrow U x, U y>U x} v(U y)=v\left((U x)_{+}\right)=v(U x)
$$

(последнее равенство в силу определения $M_{2}(G)$ ). Иными словами, выполнено (21). Согласно (21), при каждом $r \in I \quad v_{k}(x) \in M_{2}(G)$. Покажем, что для каждого $x \in G$ существуют такие $k_{x} \in I$ и $c_{x}<\infty$, что при $k>k_{x}, k \in I$

$$
v_{k}(x) \leqslant c_{x}
$$


В самом деле, для произвольного замкнутого шара $A$ с вершиной в точке $x$ такого, что $A \subset G$ и $\mu(\partial A)=0$, согласно (19) и (17),

$$
\mu(A)=\lim _{k \rightarrow \infty, k \in I} \int_{A} u_{k}(y) v_{k}(y) d y .
$$

Стало быть, существует $k_{1 x} \in I$ такое, что для всех $k>k_{1 x}, k \in I$,

$$
\int_{A} u_{k}(y) v_{k}(y) d y<\mu(A)+1 \text {. }
$$

Однако

$$
\int_{A} u_{k}(y) v_{k}(y) d y \geqslant \inf _{y \in A} u_{k}(y) \int_{B} v_{k}(y) d y \geqslant \inf _{y \in A} u_{k}(y)|B| v_{k}(x),
$$

где $B=\{y: y \in A, y \stackrel{\Gamma}{<} x\}$ в случае, когда $v$ не возрастает, $B=\{y: y \in A$, $y \stackrel{\Gamma}{>} x\}$ в случае, когда $v$ не убывает. Пусть $H_{e}(U, G)=\phi_{1}$. Так как $u \in R(U, G)$, то

$$
u_{k}(y) \stackrel{y \in A}{\rightrightarrows} \phi_{1}(y)>0 \quad(k \rightarrow \infty, k \in I) .
$$

Стало быть, существует $k_{x}>k_{1 x}, k_{x} \in I$, такое, что при $k>k_{x}, k \in I$

$$
\inf _{y \in A} u_{k}(y) \geqslant b>0 \text {. }
$$

Из неравенств (23)-(25) следует неравенство $(22)$ с $c_{x}=(\mu(A)+1) /(|B| b)$. По лемме 2 множество функций $\left\{v_{k}(x), k>k_{x}, k \in I\right\}$ слабо предкомпактно. Пусть для некоторого неограниченного множества $J \subseteq I$

$$
v_{k} \Rightarrow h \quad(k \rightarrow \infty, k \in J) .
$$

Покажем, что функция $\phi_{1}(x) h(x)$ есть плотность меры $\mu$ по мере Лебега в $G$. Пусть множество $A \in \mathfrak{A}, \bar{A}=A \cup \partial A \subset G$. Тогда существуют такие $\varepsilon>0$ и $\lambda>0$, что для произвольных $x \in A$ имеем $\varepsilon e \stackrel{\Gamma}{<} x \stackrel{\Gamma}{<} \lambda e$. Так как $u \in R(U, G)$, то найдутся такие $k_{0}$ и $c<\infty$, что при $k>k_{0}, k \in I$ и $x \in \bar{A}$

$$
\frac{u\left(U_{k} x\right)}{u\left(U_{k} e\right)} \leqslant c .
$$

Согласно (22) и (27), при $k>\max \left(k_{0}, k_{\varepsilon e}, k_{\lambda e}\right), k \in I$ и $x \in A$

$$
\frac{f\left(U_{k} x\right)}{\rho_{k}}=\frac{u\left(U_{k} x\right)}{u\left(U_{k} e\right)} v_{k}(x) \leqslant c \max \left(c_{\varepsilon e}, c_{\lambda e}\right) .
$$

По теореме Лебега в силу (26) и (28)

$$
\int_{A} \frac{f\left(U_{k} x\right)}{\rho_{k}} d x \rightarrow \int_{A} \phi_{1}(x) h(x) d x \quad(k \rightarrow \infty, k \in J) .
$$

Допустим, что $\mu(\partial A)=0$. Тогда наряду с (29) выполнено также соотношение

$$
\mu_{k}(A)=\int_{A} \frac{f\left(U_{k} x\right)}{\rho_{k}} d x \rightarrow \mu(A) \quad(k \rightarrow \infty, k \in I)
$$


(см. (19)). Из (29) и (30) следует, что

$$
\int_{A} \phi_{1}(x) h(x) d x=\mu(A) .
$$

Согласно последнему равенству, функция $h$ определяется по $\mu$ однозначно с точностью до множества лебеговой меры нуль. Однако в силу (26), $h \in M(G)$; следовательно, для каждого $x \in G$ должно быть $h(x)=\lim _{y \downarrow x} h(y)$, так что $h$ определяется по $\mu$ однозначно. Поскольку предельная функция в (26) единственна, то на самом деле

$$
v_{k} \Rightarrow h \quad(k \rightarrow \infty, k \in I) .
$$

Стало быть, в каждой точке $x$ непрерывности функции $h$ мы имеем:

$$
\frac{f\left(U_{k} x\right)}{\rho_{k}}=\frac{u\left(U_{k} x\right)}{u\left(U_{k} e\right)} v_{k}(x) \longrightarrow \phi_{1}(x) h(x) \quad(k \rightarrow \infty, k \in I),
$$

причем выполнено соотношение (31). Из (33) вытекает, что выполнено соотношение (13) с $\phi(x)=\phi_{1}(x) h(x)$. Из (31) следует, что $\phi=\phi_{1} h$ есть плотность меры $\mu$ по мере Лебега в $G$. Наконец, из соотношений (18) и (19) следует (14).

Теперь предположим, что $\phi(x)$ непрерывна в $G$. Остается доказать, что соотношение (13) выполнено равномерно по $x \in K$ для произвольного компакта $K \subset G$. Согласно теореме 2 из [18], достаточно показать, что для произвольного $x \in G$ при $x_{k} \in G, x_{k} \rightarrow x(k \rightarrow \infty, k \in I)$

$$
\frac{f\left(U_{k} x_{k}\right)}{\rho_{k}} \rightarrow \phi(x) \quad(k \rightarrow \infty, k \in I) .
$$

Так как $\phi(x)$ непрерывна в $G$ и $\phi_{1}(x)$ положительна и непрерывна в $G$, то и функция $h(x)$ непрерывна в $G$. Зафиксируем некоторые числа $a \in(0,1)$ и $b \in(1, \infty)$. Предположим для определенности, что $h \in M_{2}(G)$. Тогда справедливы следуюшие неравенства:

$$
\begin{aligned}
\limsup _{k \rightarrow \infty, k \in I} \frac{f\left(U_{k} x_{k}\right)}{\rho_{k}} & =\limsup _{k \rightarrow \infty, k \in I} \frac{u\left(U_{k} x_{k}\right)}{u\left(U_{k} e\right)} v_{k}\left(x_{k}\right) \\
& \leqslant \lim _{k \rightarrow \infty, k \in I} \frac{u\left(U_{k} x_{k}\right)}{u\left(U_{k} e\right)} v_{k}(a x)=\phi_{1}(x) h(a x)
\end{aligned}
$$

(последнее равенство следует из (3) и (32) с учетом того, что $h$ непрерывна в $a x)$. Переходя в (35) к пределу по $a \uparrow 1$, получаем с учетом непрерывности $h$ в точке $x$, что

$$
\limsup _{k \rightarrow \infty, k \in I} \frac{f\left(U_{k} x_{k}\right)}{\rho_{k}} \leqslant \phi_{1}(x) h(x) .
$$

Далее, мы имеем оценку снизу:

$$
\begin{aligned}
\liminf _{k \rightarrow \infty, k \in I} \frac{f\left(U_{k} x_{k}\right)}{\rho_{k}} & =\liminf _{k \rightarrow \infty, k \in I} \frac{u\left(U_{k} x_{k}\right)}{u\left(U_{k} e\right)} v_{k}\left(x_{k}\right) \\
& \geqslant \lim _{k \rightarrow \infty, k \in I} \frac{u\left(U_{k} x_{k}\right)}{u\left(U_{k} e\right)} v_{k}(b x)=\phi_{1}(x) h(b x) .
\end{aligned}
$$


Переходя в (37) к пределу по $b \downarrow 1$, получаем, что

$$
\liminf _{k \rightarrow \infty, k \in I} \frac{f\left(U_{k} x_{k}\right)}{\rho_{k}} \geqslant \phi_{1}(x) h(x) .
$$

Из (36) и (38) следует (34) с $\phi(x)=\phi_{1}(x) h(x)$. В случае, когда $h \in M_{1}(G)$, (34) доказывается точно так же, только неравенства заменятся на противоположные, a limsup и $\lim$ inf поменяются местами. Доказательство теоремы завершено.

Пусть, как и ранее, $\Gamma$ - произвольный замкнутый выпуклый острый телесный конус, допускающий семейство линейных преобразований $U=\left\{U_{k}, k \in I\right\}$, которые оставляют $\Gamma$ инвариантным. Справедлива следуюшая тауберова теорема сравнения.

Теорема 2. Пусть $f(x)=u(x) v(x)(x \in G)$, где $u(x) \in R(U, G)$ (см. определение 2$), v(x) \in M(G)$ (см. обозначение 3 после леммы 1 ), $v(x) \geqslant 0(x \in G)$ u для всех $y \in \mathbf{C}$ существует $\widehat{f}(y)$. Предположим, что функиия $g(x)$ вполне допустима для семейства $U=\left\{U_{k}, k \in I\right\}$ (см. определение 3). Если для произвольного у $\in \mathbf{C}$

$$
\frac{\widehat{f}\left(V_{k} y\right)}{\widehat{g}\left(V_{k} y\right)} \rightarrow 1 \quad(k \rightarrow \infty, k \in I)
$$

$\left(V_{k}=\left(U_{k}^{*}\right)^{-1}\right)$, то сущестөует предел

$$
\frac{f\left(U_{k} x\right)}{g\left(U_{k} x\right)} \stackrel{x \in K}{\rightrightarrows} 1 \quad(k \rightarrow \infty, k \in I)
$$

для произвольного компакта $K \subset G$.

Д о к а з а т е л с т в о. В силу вполне допустимости функции $g$ для семейства $U=\left\{U_{k}, k \in I\right\}$ для некоторого фиксированного вектора $e \in G$

$$
\frac{g\left(U_{k} x\right)}{g\left(U_{k} e\right)} \stackrel{x \in K}{\rightrightarrows} \phi(x)>0 \quad(k \rightarrow \infty, k \in I)
$$

для произвольного компакта $K \subset G$. Положим $\rho_{k}=g\left(U_{k} e\right), k \in I$. Покажем, что

$$
\frac{f\left(U_{k} x\right)}{\rho_{k}} \stackrel{x \in K}{\rightrightarrows} \phi(x) \quad(k \rightarrow \infty, k \in I)
$$

для произвольного компакта $K \subset G$. В самом деле, при $y \in \mathbf{C}$

$$
\frac{\widehat{f}\left(V_{k} y\right)}{J_{k} \rho_{k}}=\frac{\widehat{f}\left(V_{k} y\right)}{\widehat{g}\left(V_{k} y\right)} \frac{\widehat{g}\left(V_{k} y\right)}{J_{k} g\left(U_{k} e\right)},
$$

причем по теореме Лебега в силу (41) и оценки вида (5) для функции $g(x)$

$$
\frac{\widehat{g}\left(V_{k} y\right)}{J_{k} g\left(U_{k} e\right)}=\int_{G} \frac{g\left(U_{k} x\right)}{g\left(U_{k} e\right)} e^{-(y, x)} d x \rightarrow \widehat{\phi}(y)<\infty
$$


при $k \rightarrow \infty, k \in I$ для произвольного $y \in \mathbf{C}$. Поэтому из (39) и (43) следует, что $\hat{f}\left(V_{k} y\right) /\left(J_{k} \rho_{k}\right) \rightarrow \hat{\phi}(y)<\infty(k \rightarrow \infty, k \in I) \forall y \in \mathbf{C}$. Стало быть, выполнены все предположения теоремы 1 с $\psi(y)=\widehat{\phi}(y)$. Поскольку функция $\phi(x)$ непрерывна в $G$, то, пользуясь справедливостью теоремы 1 , убеждаемся в том, что выполнено соотношение (42). Заметим, что

$$
\frac{f\left(U_{k} x\right)}{g\left(U_{k} x\right)}=\frac{f\left(U_{k} x\right)}{\rho_{k}}\left(\frac{g\left(U_{k} x\right)}{g\left(U_{k} e\right)}\right)^{-1} .
$$

Из соотношений $(41),(42)$ и (45) в силу положительности $\phi(x)$ следует (40). Теорема 2 доказана.

Пусть $\Delta_{\Gamma}(x)$ есть расстояние от точки $x \in \Gamma$ до границы конуса $\Gamma$ $\left(\Delta_{T}(x)\right.$ есть расстояние от точки $x \in T$ до границы конуса $\left.T=\Gamma^{*}\right)$.

О п р е д е ле н и е 6 . Выпуклый конус $\Gamma$ называется однородным, если для произвольных точек $x_{1}$ и $x_{2}$ из $G=\operatorname{int} \Gamma$ существует невырожденное линейное преобразование $U$ (вообще говоря, зависящее от $x_{1}$ и $x_{2}$ ), оставляющее конус $\Gamma$ инвариантным, такое, что $U x_{1}=x_{2}$.

Пусть $\Gamma$ - произвольный замкнутый выпуклый острый телесный однородный конус в $\mathbf{R}^{n}$ с вершиной в нуле. Справедлива следующая тауберова теорема.

Теорема 3. Пусть $f(x)=u(x) v(x)(x \in G)$, причем $v(x) \in M(G)$ (см. обозначение 3 после леммы 1$), v(x) \geqslant 0(x \in G) u$ для всех $y \in \mathbf{C}$ суиествует $\widehat{f}(y)$. Справедливы следующие утверждения.

1. Если $g(x) \in D_{1}(\Gamma)$ (см. определение 5$), u(x) \in R_{1}(\Gamma)$ (см. обозначение 1) и существует предел

mo

$$
\frac{\widehat{f}(y)}{\widehat{g}(y)} \rightarrow 1 \quad(y \rightarrow 0, y \in \mathbf{C})
$$

$$
\frac{f(x)}{g(x)} \rightarrow 1 \quad\left(x \in G, \Delta_{\Gamma}(x) \rightarrow \infty\right) .
$$

2. Eсли $g(x) \in D_{2}(\Gamma)$ (см. определение 5$), u(x) \in R_{2}(\Gamma)$ (см. обозначение 1) и для произвольного $b>0$ существует предел

$$
\frac{\widehat{f}(y)}{\widehat{g}(y)} \rightarrow 1 \quad\left(\Delta_{T}(y) \rightarrow 0, y \in \mathbf{C},|y|<b\right)
$$

то для произвольного $\delta>0$

$$
\frac{f(x)}{g(x)} \rightarrow 1 \quad\left(x \in G, \Delta_{\Gamma}(x) \geqslant \delta\right) .
$$

3. $\operatorname{Eсли~} g(x) \in D_{3}(\Gamma)$ (см. определение 5$), u(x) \in R_{3}(\Gamma)$ (см. обозначение 1) и существует предел

$$
\frac{\widehat{f}(y)}{\widehat{g}(y)} \rightarrow 1 \quad\left(\Delta_{T}(y) \rightarrow 0, y \in \mathbf{C}\right)
$$


mo

$$
\frac{f(x)}{g(x)} \rightarrow 1 \quad(x \in G,|x| \rightarrow \infty) .
$$

Д ок а з т е л ь с т о. Докажем сначала п. 1. Предположим противное, т.е. пусть (46) выполнено, а (47) не имеет места. Тогда существуют $\varepsilon>0$ и последовательность $x_{k} \in G, \Delta_{\Gamma}\left(x_{k}\right) \geqslant k(k \in \mathbf{N})$, такие, чTO

$$
\left|\frac{f\left(x_{k}\right)}{g\left(x_{k}\right)}-1\right|>\varepsilon \quad(k \in \mathbf{N}) .
$$

Зафиксируем $a \in G,|a|=1$, и рассмотрим семейство линейных операторов $U=\left\{U_{k}, k \in \mathbf{N}\right\}, U_{k} \Gamma=\Gamma$, таких, что $U_{k} a=x_{k}$. Такие операторы существуют в силу однородности $\Gamma$. Так как $\left|U_{k} a\right|=\left|x_{k}\right| \geqslant k$, то

$$
k \leqslant \Delta_{\Gamma}\left(x_{k}\right)=\Delta_{\Gamma}\left(U_{k} a\right)=\frac{\lambda(k)}{p(a)},
$$

где $\lambda(k)$ задаются соотношением (2), а $p(a)>0$ (см. лемму 2 из [5, гл. II, раздел 5.1]). Стало быть, $\lambda(k) \rightarrow \infty(k \rightarrow \infty, k \in \mathbf{N})$, а следовательно, и $\Lambda(k) \rightarrow \infty(k \rightarrow \infty, k \in \mathbf{N})$ (см. (2)). Таким образом, семейство $\left\{U_{k}, k \in \mathbf{N}\right\}$ будет семейством первого типа, а так как $g(x) \in D_{1}(\Gamma)$, то существует подсемейство $\left\{U_{k}, k \in L \subseteq \mathbf{N}\right\}(L-$ неограничено), относительно которого $g(x)$ будет вполне допустимой. В силу определения $R_{1}(\Gamma)$ (см. обозначение 1 ) в семействе $\left\{U_{k}, k \in L\right\}$ найдется такое подсемейство $U=\left\{U_{k}, k \in M \subseteq L\right\}$ ( $M$ - неограничено), что $u \in R(U, \Gamma)$. Ясно, что функция $g(x)$ останется вполне допустимой и относительно семейства $U^{r}$. Для произвольного фиксированного вектора $y \in \mathbf{C}$ и $k \in \mathbf{N} V_{k} y \in \mathbf{C}_{\text {и }}$

$$
\left|V_{k} y\right| \leqslant|y| \sup _{|e|=1}\left|V_{k} e\right|=\frac{|y|}{\lambda(k)} \longrightarrow 0 \quad(k \rightarrow \infty, k \in \mathbf{N}) \text {. }
$$

Поэтому по условию (46)

$$
\frac{\widehat{f}\left(V_{k} y\right)}{\widehat{g}\left(V_{k} y\right)} \rightarrow 1 \quad(k \rightarrow \infty, k \in M) .
$$

Стало быть, выполнены все условия теоремы 2. Согласно этой теореме,

$$
\frac{f\left(U_{k} a\right)}{g\left(U_{k} a\right)}=\frac{f\left(x_{k}\right)}{g\left(x_{k}\right)} \rightarrow 1 \quad(k \rightarrow \infty, k \in M) .
$$

Последнее соотношение противоречит неравенству (52) для $k \in M$. Пункт 1 теоремы 3 доказан. Доказательство п. 2 (соответственно 3) происходит при помощи дословного повторения предыдущих рассуждений с учетом того, что в указанных случаях семейство операторов $\left\{U_{k}, k \in \mathbf{N}\right\}$ будет семейством 2-го (соответственно 3-го) типа (см. [5, гл. II, раздел 6.2, теорема 2]). 
3. Асимптотика безгранично делимых распределений в конусе. Пусть $\Gamma$ - произвольный замкнутый выпуклый острый телесный однородный конус в $\mathbf{R}^{n}$ с вершиной в нуле. При $x \in \Gamma$ положим

$$
\begin{gathered}
\Gamma(x)=\{y: y \in \Gamma, x-y \in \Gamma\}=\{y: y \leqslant x\}, \\
\tilde{\Gamma}(x)=\Gamma \backslash \Gamma(x) .
\end{gathered}
$$

Предположим, что случайный вектор $\xi \in \mathbf{R}^{n}$ имеет безгранично делимое распределение, сосредоточенное в $Г$, причем его преобразование Лапласа имеет вид:

$$
\mathbf{E} e^{-(\lambda, \xi)}=\exp \left\{-\int_{\Gamma}\left(1-e^{-(\lambda, x)}\right) \nu(d x)\right\} \quad\left(\lambda \in \Gamma^{*}\right),
$$

где $\nu$ - $\sigma$-конечная мера на $\Gamma$ (возможно, неограниченная в окрестности нуля), для которой выполнены соотношения $\int_{|x| \leqslant 1}|x| \nu(d x)<\infty$, $\int_{|x|>1} \nu(d x)<\infty$ (см. [21, лемма 3]). Мера $\nu$ называется спектральной мерой Леви распределения случайного вектора $\xi$. Положим при $x \in G$

$$
f(x)=\mathbf{P}\{\xi \in \widetilde{\Gamma}(x)\}, \quad g(x)=\nu(\widetilde{\Gamma}(x)) .
$$

Справедлива следующая предельная теорема.

Теорема 4. Пусть функиия $g(x)$ является допустимой функиией первого типа для конуса $\Gamma\left(g(x) \in D_{1}(\Gamma)\right)$ - см. определение 5. Тогда

$$
f(x)=(1+o(1)) g(x) \quad\left(x \in G, \Delta_{\Gamma}(x) \rightarrow \infty\right),
$$

m.e. $n p u x \in G=\operatorname{int} \Gamma, \Delta_{\Gamma}(x) \rightarrow \infty$

$$
\mathbf{P}\{\xi \in \tilde{\Gamma}(x)\}=(1+o(1)) \nu(\widetilde{\Gamma}(x)),
$$

где $\Delta_{\Gamma}(x)$ есть расстояние от точки $x$ до границы конуса $\Gamma$.

3. а м е ч а н и е 5 . Теорема 4 обобщает соответствуюшие утверждения из работ [20], [21]. Отметим, что в цитируемых работах $\Delta_{\Gamma}(x)$ имела порядок $|x|$ при $|x| \rightarrow \infty$; здесь же допускается сколь угодно медленное стремление $\Delta_{\Gamma}(x)$ к бесконечности.

Теорема 4 будет доказана как следствие п. 1 тауберовой теоремы 3. Заманчиво было бы, воспользовавшись п. 2 (соответственно 3) доказать, что (57) имеет место при $|x| \rightarrow \infty$ и $\Delta_{\Gamma}(x) \geqslant \delta>0$ (соответственно $|x| \rightarrow \infty$ и $x \in G)$. Однако следующие простые соображения показывают, что (57), вообще говоря, не выполнено уже при $|x| \rightarrow \infty$ и $\Delta_{\Gamma}(x) \geqslant \delta>0$. В самом деле, пусть $\Gamma=\mathbf{R}_{+}^{n}=\left\{x=\left(x_{1}, \ldots, x_{n}\right), x_{i} \geqslant 0 \forall i=1, \ldots, n\right\}$, и пусть $|x| \rightarrow \infty$ так, что $x_{1}=\delta>0, \min \left(x_{2}, \ldots, x_{n}\right) \rightarrow \infty$. Тогда $g(x) \rightarrow \nu(A)$, где $A=\left\{y: y=\left(y_{1}, \ldots, y_{n}\right) \in \mathbf{R}_{+}^{n}, y_{1}>\delta\right\}$, и если $\nu(A)>1$, то не может иметь места (57), так как $f(x)=\mathbf{P}\{\xi \in \widetilde{\Gamma}(x)\} \leqslant 1$.

В качестве примеров $g(x)$ мы можем привести примеры 1-3 в дополнительном предположении, что $g(x)$ не возрастает в $\Gamma$ (см. п. 2). 
Положим

$\theta_{\Gamma}(x)=\left\{\begin{array}{ll}1, & x \in \Gamma, \\ 0, & x \notin \Gamma,\end{array} \quad K_{\mathbf{C}}(\lambda)=\widehat{\theta}_{\Gamma}(\lambda)=\int_{\Gamma} e^{-(\lambda, x)} d x \quad(\lambda \in \mathbf{C})\right.$, $\nu(x)=\nu\{y: y \stackrel{\Gamma}{\leqslant} x\}$. Теорему 4 мы докажем при помощи следующей леммы.

Лемма 4. Для произвольного $\lambda \in \mathbf{C}$ выполнено соотношение

$$
K_{\mathbf{C}}(\lambda) \int_{\Gamma}\left(1-e^{-(\lambda, x)}\right) \nu(d x)=\int_{\Gamma} e^{-(\lambda, x)} g(x) d x<\infty .
$$

Предположим сначала, что $\nu(\Gamma)<\infty$. Мы имеем при $\lambda \in \mathbf{C}$

$$
\int_{\Gamma} e^{-(\lambda, x)} \nu(x) d x=\int_{\Gamma} e^{-(\lambda, x)}\left(\nu * \theta_{\Gamma}\right)(x) d x=\int_{\Gamma} e^{-(\lambda, x)} \nu(d x) \cdot K_{\mathbf{C}}(\lambda) .
$$

Ho $g(x)=\nu(\Gamma)-\nu(x)$, поэтому $\int_{\Gamma} e^{-(\lambda, x)} \nu(x) d x=\nu(\Gamma) K_{\mathbf{C}}(\lambda)-\widehat{g}(\lambda)$. Стало быть,

$$
\begin{aligned}
\widehat{g}(\lambda) & =\nu(\Gamma) K_{\mathbf{C}}(\lambda)-\int_{\Gamma} e^{-(\lambda, x)} \nu(d x) \cdot K_{\mathbf{C}}(\lambda) \\
& =\int_{\Gamma}\left(1-e^{-(\lambda, x)}\right) \nu(d x) \cdot K_{\mathbf{C}}(\lambda) \quad \forall \lambda \in \mathbf{C},
\end{aligned}
$$

откуда следует $(58)$ в случае, когда $\nu(\Gamma)<\infty$. В общем случае положим

$$
\Gamma_{k}=\left\{u: u \in \Gamma,|u| \geqslant k^{-1}\right\}, \quad \nu_{k}(A)=\nu\left(A \cap \Gamma_{k}\right), \quad g_{k}(x)=\nu_{k}(\widetilde{\Gamma}(x))
$$

для произвольных $A \in \mathfrak{A}, x \in \Gamma, k \in \mathbf{N}$. Так как для мер $\nu_{k}$ лемма уже доказана, то для каждого $\lambda \in \mathbf{C}$

$$
\widehat{g}_{k}(\lambda)=K_{\mathbf{C}}(\lambda) \int_{\Gamma_{k}}\left(1-e^{-(\lambda, x)}\right) \nu(d x) .
$$

Поэтому для всех $\lambda \in \mathbf{C}$ при $k \rightarrow \infty$

$$
\widehat{g}_{k}(\lambda) \longrightarrow K_{\mathbf{C}}(\lambda) \int_{\Gamma}\left(1-e^{-(\lambda, x)}\right) \nu(d x),
$$

так как $\int_{\Gamma}\left(1-e^{-(\lambda, x)}\right) \nu(d x)<\infty$ при $\lambda \in \mathbf{C}$. Но, поскольку для произвольных $x \in G$ и $\lambda \in \mathbf{C}$ при $k \rightarrow \infty$

$$
e^{-(\lambda, x)} g_{k}(x) \uparrow e^{-(\lambda, x)} g(x),
$$

то по теореме Б. Леви при $k \rightarrow \infty$

$$
\widehat{g}_{k}(\lambda)=\int_{\Gamma} e^{-(\lambda, x)} g_{k}(x) d x \rightarrow \int_{\Gamma} e^{-(\lambda, x)} g(x) d x<\infty
$$

для всех $\lambda \in \mathbf{C}$. Сравнивая (59) и (60), получаем (58). Лемма доказана. Док аз ат ельст во те о ремы 4. Из (55) и (58) следует, что

$$
\mathbf{E} e^{-(\lambda, \xi)}=\exp \left\{-\frac{\widehat{g}(\lambda)}{K_{\mathbf{C}}(\lambda)}\right\} \quad \forall \lambda \in \mathbf{C}
$$


Пусть $P$ - распределение случайного вектора $\xi$. Точно так же, как и в (58), мы устанавливаем, что

$$
1-\tilde{P}(\lambda)=\int_{\Gamma}\left(1-e^{-(\lambda, x)}\right) P(d x)=\frac{\widehat{f}(\lambda)}{K_{\mathbf{C}}(\lambda)} \quad \forall \lambda \in \mathbf{C} .
$$

Из (61) и (62) следует, что

$$
\frac{\widehat{f}(\lambda)}{K_{\mathbf{C}}(\lambda)}=1-\exp \left\{-\frac{\widehat{g}(\lambda)}{K_{\mathbf{C}}(\lambda)}\right\} \quad \forall \lambda \in \mathbf{C} .
$$

При $\lambda \in \mathbf{C}$ положим $\zeta_{\lambda}=(\lambda, \xi)$. Мы имеем:

$$
\zeta_{\lambda} \geqslant 0 \quad \forall \lambda \in \mathbf{C}, \quad \zeta_{\lambda} \stackrel{P}{\rightarrow} 0 \quad(\lambda \rightarrow 0, \lambda \in \mathbf{C}) .
$$

Так как функция $\exp \{-t\}$ при $t \geqslant 0$ непрерывна и ограничена, то

$$
\mathbf{E} e^{-(\lambda, \xi)}=\mathbf{E} e^{-\zeta_{\lambda}} \longrightarrow 1 \quad(\lambda \rightarrow 0, \lambda \in \mathbf{C}) .
$$

Из (61) и (64) следует, что

$$
\frac{\widehat{g}(\lambda)}{K_{\mathbf{C}}(\lambda)}=-\ln \mathbf{E} e^{-(\lambda, \boldsymbol{\xi})} \rightarrow 0 \quad(\lambda \rightarrow 0, \lambda \in \mathbf{C}) .
$$

Из (63) и (65) получаем, что

$$
\frac{\widehat{f}(\lambda)}{K_{\mathbf{C}}(\lambda)}=(1+o(1)) \frac{\widehat{g}(\lambda)}{K_{\mathbf{C}}(\lambda)} \quad(\lambda \rightarrow 0, \lambda \in \mathbf{C}),
$$

или же, что $\widehat{f}(\lambda)=(1+o(1)) \hat{g}(\lambda)(\lambda \rightarrow 0, \lambda \in \mathbf{C})$. Мы видим, что выполнены все предположения п. 1 теоремы 3 . Воспользовавшись этой теоремой, убеждаемся в справедливости соотношения (57). Теорема доказана.

\section{СПИСОК ЛИТЕРАТУРЫ}

1. Антонов С. Н. К асимптотическому поведению безгранично делимых законов. Матем. заметки, 1980, т. 28, №6, с. 939-946.

2. Антонов С. Н., Круглов В. М. Об асимптотическом поведении безгранично делимых распределений в банаховом пространстве. - Теория вероятн. и ее примен., 1982 , т. 27 , в. 4 , с. $625-642$.

3. Антонов С.Н., Круглов В.М. Еще раз об асимптотическом поведении безгранично делимых распределений в банаховом пространстве. - Теория вероятн. и ее примен., 1984 , т. 29 , в. 4 , с. $735-742$.

4. Владимиров В.С. Многомерное обобщение тауберовой теоремы Харди и Литтлвуда. - Изв. АН СССР, сер. матем., 1976, т. 40, №5, с. 1084-1101.

5. Владимиров В.С., Дрожжинов Ю. Н., Завьялов Б. И. Многомерные тауберовы теоремы для обобщенных функций. М.: Наука, 1986, 304 с.

6. Дрожжинов Ю. Н., Завьялов Б. И. Теоремы сравнения тауберова типа. - Докл. AH CCCP, 1984, т. 279, № 3, c. 532-535.

7. Дрожжинов Ю. Н., Завьялов Б.И. Многомерные тауберовы теоремы сравнения для обобщенных функций в конусах. - Матем. сб., 1985, т. 126, № 4, с. 515-542. 
8. Круглов В. М. Замечание к теории безгранично делимых законов. - Теория вероятн. и ее примен., 1970 , т. 15 , в. 2 , с. $330-336$.

9. Круглов В.М. Интегралы по безгранично делимым распределениям в гильбертовом пространстве. - Матем. заметки, 1972, т. 11, № 6, с. 669-676.

10. Круглов В.М. Сходимость числовых характеристик сумм независимых случайных величин со значениями в гильбертовом пространстве. - Теория вероятн. и ее примен., 1973, т. 18, № 4, с. 734-752.

11. Круглов В. М. О безгранично делимых распределениях в гильбертовом пространстве. - Матем. заметки, 1974, т. 16, № 4, с. 585-594.

12. Круглов В. М. Характеризация одного класса распределений в гильбертовом пространстве. - Матем. заметки, 1974, т. 16, № 5, с. 777-782.

13. Круглов В. М. Дополнительные главы теории вероятностей. Учеб. пособие. М.: Высшая школа, 1984, $264 \mathrm{c.}$

14. Сәибнев $M$. C. Асимптотика безгранично делимых распределений в $\mathbf{R}^{n}$. - Труды Ин-та математики СО АН СССР, 1989, т. 13, с. 100-116.

15. Улановский $A . M$. Об асимптотическом поведении безгранично делимых распределений на бесконечности. - Теория функций, функциональный анализ и их приложения, 1981, № 35, с. 100-107.

16. Якьмив А. Л. Предельные теоремы для ветвящихся процессов. Дисс.... канд. физ.матем. наук. М.: МГУ, 1981.

17. Якьимив $A$. Л. Многомерные тауберовы теоремы и их применение к ветвящимся процессам Беллмана-Харриса. - Матем. с6., 1981, т. 115, № 3, с. 463-477.

18. Якьмие А.Л. Асимптотика вероятности продолжения критических ветвящихся процессов Беллмана-Харриса. - Труды МИАН, 1986, т. 177, с. 177-205.

19. Sato Ken-Iti. Lévy Processes and Infinitely Divisible Distributions. Cambridge: Cambridge Univ. Press, 1999, 486 p.

20. Omey E. Infinite divisibility and random sums of random vectors. - Yokohama Math. J., 1985 , v. 33, № 1-2, p. 39-48.

21. Yakymiv A. L. The asymptotics of multidimensional infinite divisible distributions. J. Math. Sci., (New York), 1997, v. 84, № 3, p. 1197-1207.

Поступила в редакцию

5.XII. 2002 